\title{
A construção de um instrumento de avaliação discente de um programa de pós-graduação
}

\author{
Lilian Milnitsky Stein ${ }^{1}$ \\ Denise Falcke \\ Juliana Carmona Predebon \\ Kátia Bones Rocha \\ Luciana Moreira de Avila \\ Mariana Porto Ruwer de Azambuja
}

\begin{abstract}
Resumo
O artigo apresenta o processo de construção e validação de conteúdo e aparente de um instrumento de avaliação discente para programas de pós-graduação. Para este processo foram desenvolvidas diversas etapas envolvendo tanto uma amostra de 14 docentes de um programa de pós-graduação de Psicologia, quanto a testagem piloto numa amostra de 52 pós-graduandos. O instrumento assim aprimorado foi testado numa nova amostra de 63 estudantes (46 mestrandos e 17 doutorandos). Os resultados, tanto quantitativos quanto qualitativos, indicaram que o instrumento possui bons indicadores de consistência interna para a escala como um todo $($ alpha $=0,9332)$ e para suas subescalas, como também de validade de conteúdo e aparente. Espera-se com este trabalho poder contribuir para a avaliação de outros programas de pós-graduação e auxiliar demais pesquisadores na construção de seus instrumentos de pesquisa.

Palavras-Chave: Avaliação discente; Pós-Graduação; Construção de instrumentos.
\end{abstract}

\section{Development of an instrument for students' evaluation of a graduate program}

\begin{abstract}
This paper presents the process of developing an instrument for students' assessment of a Graduate Program. That process encompassed a series of steps involving a sample of 14 faculty members of a Psychology Graduate Program as well as a pilot sample of 52 graduate students. The revised instrument was tested on a group of 62 students (46 masters and 17 doctoral students). Both quantitative and qualitative results indicated that the instrument was reliable with high internal consistency as a whole $(\mathrm{alpha}=0,9332)$ and as sub-scales. In addition, those results also suggested that the instrument was valid (face and content validity). We hope to have contributed with this work to other graduate programs in their student assessment system and also to researchers interested in scale development. Keywords: Student evaluation; Graduate education; Scale development.
\end{abstract}

\section{Introdução}

O Brasil possui um sistema universitário relativamente jovem, sobretudo com relação à pós-graduação. Com o franco desenvolvimento observado na última década, especialmente no setor privado, e com a aproximação da marca de dois milhões e meio de estudantes matriculados no Ensino Superior, estamos prestes a alcançar o estágio já atingido por alguns países inclusive latino-americanos - o qual se convencionou chamar de "educação superior de massa". Os altos índices de expansão, geralmente aliados à busca de uma maior diversificação e introdução de inovações, tendem a ser acompanhados pelo receio de que a qualidade da formação esteja ameaçada. Uma das alternativas de controle da qualidade de ensino é a construção de um sistema de avaliação preciso (Spagnolo, 2000). Both (1997) aponta que o processo de avaliação institucional das universidades é um agente indispensável e perene de modernização e de elevação do nível de desempenho administrativo e educacional.

Com relação à avaliação institucional, Pereira (1997) destaca a necessidade de as universidades, ao se conscientizarem da importância da realização da avaliação institucional, terem claro os objetivos que pretendem alcançar. Ressalta, como aspecto fundamental, que as peculiaridades da própria instituição, assim como as características locais, regionais e até mesmo nacionais,

${ }^{1}$ Endereço para correspondência:

Programa de Pós-Graduação em Psicologia da PUCRS, Porto Alegre

Av. Ipiranga, 6.681 - Prédio 11 - 9o andar - 90619-900 - Porto Alegre-RS

Fone/Fax: (51) 3320-3633

E-mail: lilian@pucrs.br 
sejam contempladas. Além disso, afirma que a avaliação institucional deve ser um processo contínuo de busca por melhoria da qualidade das atividades desenvolvidas. Both (1997), por sua vez, pontua ainda que cabe às instituições a autonomia, tanto no apontamento da filosofia, da política, dos objetivos, dos métodos, dos componentes de avaliação quanto do destino a ser dado aos resultados obtidos por meio do processo avaliativo.

A formalização de um processo de avaliação institucional que hoje tomou novo impulso mediante as práticas dimensionadas no Ministério da Educação pelo INEP (Instituto Nacional de Estudos e Pesquisas Educacionais) através do SINAES (Sistema Nacional de Avaliação do Ensino Superior). Neste aspecto, cabe ressaltar que, no Brasil, a Coordenação de Aperfeiçoamento de Pessoal de Nível Superior (CAPES) foi uma das pioneiras na implementação de um sistema de avaliação do ensino superior, particularmente neste caso da pós-graduação, mesmo quando a maior parte dos programas de pós-graduação hoje existentes ainda não havia se estabelecido (Spagnolo, 2000).

O sistema avaliativo da CAPES, desde sua implantação, vem sofrendo mudanças buscando seu aperfeiçoamento. No ano de 1996, foi constatado que os dados obtidos pela antiga forma de avaliação não permitiam comparações válidas entre os cursos, uma vez que todos obtinham altos graus na escala. No novo processo de avaliação da CAPES, houve mudanças em diferentes aspectos, tais como na periodicidade da avaliação (de anual para trianual), na escala de notas (de conceitos a valores numéricos) e na unidade de avaliação (que passou da avaliação de cursos para programas, os quais podem integrar mestrados e doutorados). Neste processo, o que se fez de mais importante foi introduzir, como parte fundamental da avaliação, a possibilidade de comparação entre as áreas, criando critérios de qualidade que pudessem ser homogêneos, respeitando a especificidade de cada uma (Vazquez, 2002).

Dentro deste panorama, surgiram as dimensões pelas quais hoje os programas de pós-graduação são avaliados pela CAPES. Segundo esta instituição, a pósgraduação brasileira é, atualmente, a melhor da América Latina, o que se deve, em boa medida, ao processo avaliativo por ela implementado. Contudo, isso não impede que muitas discussões venham sendo realizadas sobre o problema da qualidade comparativa entre as distintas áreas na comunidade acadêmica (Vazquez, 2002).

No que tange à proposta de um processo avaliativo mais amplo de sistemas educativos, Arreola (1995) salienta que um dos fatores fundamentais para se conquistar a credibilidade dos alunos é envolvê-los ativamente neste processo. Alinhado com a idéia de fomentar uma discussão sobre a qualidade dos cursos de mestrado e doutorado, bem como com a intenção de promover a aprendizagem do processo de construção de um instrumento de pesquisa, um grupo de alunas do Programa de Pós-Graduação em Psicologia da PUCRS, sob orientação da docente primeira autora, tomou a iniciativa de criar um instrumento que pudesse ser utilizado como uma das ferramentas para avaliar o grau de satisfação discente com o referido programa. A atividade iniciou numa disciplina optativa - ministrada pela primeira autora - e acabou tomando dimensões extracurriculares, sendo desenvolvida por mais de um ano pelo grupo de autoras. Desta forma, o presente artigo tem como objetivo primordial mostrar o processo de construção de um instrumento de pesquisa em todas as suas etapas. Como resultado, apresenta o instrumento de avaliação discente criado para o Programa de PósGraduação em Psicologia da PUCRS, tendo como parâmetros norteadores os critérios da CAPES para avaliação dos programas de pós-graduação.

\section{Método}

A pesquisa bibliográfica sobre avaliação discente de programas de pós-graduação revelou a existência de poucos materiais sobre o tema e, especialmente, a inexistência de instrumentos específicos (questionários ou escalas) elaborados para este propósito no Brasil. Diante desta limitação e perante a necessidade de elegerem-se parâmetros para a elaboração de um instrumento de avaliação discente, optou-se por utilizar as dimensões que a CAPES emprega atualmente no seu sistema de avaliação de programas de pós-graduação. Esta estratégia visou conceder maior legitimidade ao instrumento elaborado, haja vista que o parecer gerado pela CAPES, com base nesse sistema avaliativo já bem estabelecido, é um dos principais parâmetros de aferição da qualidade do ensino superior no nível da pós-graduação no país.

Em conformidade com a literatura específica da área (Anastasi \& Urbina, 2000; Devellis, 2002; Fachel \& Camey, 2000; Fink \& Kosecoff, 1998; Pasquali, 1996, 2001), o processo de construção e validação do instrumento de avaliação discente do Programa de Pós-Graduação em Psicologia da PUCRS percorreu as seguintes etapas:

\section{1a etapa - Pesquisa bibliográfica e hemerográfica em bancos de dados nacionais e internacionais}

Inicialmente, cada um dos nove alunos da disciplina de Técnicas Psicométricas do Programa de PósGraduação em Psicologia da PUCRS (no segundo semestre de 2002) realizou uma busca de material sobre instrumentos e critérios de avaliação de programas de educação superior em bancos de dados (bibliográficos e hemerográficos) nacionais e internacionais, valendo-se de sites de 
busca na internet. Constataram-se poucos registros sobre o tema, não tendo sido encontrado nenhum instrumento ou método sistemático de avaliação discente (ou mesmo, docente) de programa de pós-graduação no país.

\section{2a etapa - Definição de parâmetros de avaliação}

Tendo em vista a inexistência de uma escala ou outro tipo de ferramenta que pudesse ser adaptada para a avaliação discente de programas de pós-graduação, decidiuse construir um instrumento próprio para este fim. Optouse por adotar os parâmetros propostos pela CAPES em sua avaliação dos programas de pós-graduação no país (Vasquez, 2002). Além disso, também foram entrevistados seis alunos de mestrado e doutorado matriculados no programa, a fim de fazer-se um levantamento dos fatores que os discentes consideravam relevantes em uma avaliação desta natureza. Após as entrevistas e a análise das respostas, foram elencados os fatores propostos pelos entrevistados. Com base nestes fatores e levando-se em consideração as dimensões avaliadas pela CAPES, foram selecionadas as dimensões (apresentadas na Tabela 1) que iriam compor o instrumento de avaliação.

Tabela 1 - Dimensões do instrumento de avaliação discente do programa de pós-graduação

Dimensão Descrição

\begin{tabular}{ll}
\hline 1. Linhas de pesquisa & oo de linhas de pesquisas oferecidas e integração do projeto do respondente com o \\
projeto do professor orientador.
\end{tabular}

2. Orientador Relação entre o respondente e seu orientador: tempo, qualidade e freqüência das

3. Comunicação

4. Bolsas orientações, incentivo para a produção científica.

5. Disciplinas Com a coordenação, professores e representantes dos alunos.

Critério de concessão de bolsas.

6. Encontros científicos

Disciplinas obrigatórias e optativas freqüentadas ao longo do curso.

Temas oferecidos, palestrantes convidados, periodicidade, dia, horário, duração e obrigatoriedade do evento.

7. Cursos de extensão Temas oferecidos, número de cursos oferecidos, professores, horário, duração, custo, forma e período de divulgação.

8. Integração Integração dos alunos de graduação (bolsistas de iniciação científica) nos grupos de pesquisa, bem como no projeto de pesquisa do respondente.

9. Intercâmbio Intercâmbio entre o PPGP/PUCRS e outras unidades da PUCRS, bem como com outros cursos de pós-graduação (fora da PUCRS).

10. Biblioteca Atualidade e quantidade do acervo, qualidade e rapidez do atendimento e dos serviços de pedidos de artigos (Ligdoc).

11. Secretaria

12. Salas Atualidade, rapidez, organização e eficácia dos atendimentos.

Limpeza, iluminação, localização, computadores disponíveis nas salas dos grupos de pesquisa, nas salas de aula e nas salas dos alunos.

\section{3a etapa - Elaboração dos itens do instrumento}

Tomando por base a definição das dimensões, formaram-se subgrupos de alunos da disciplina Técnicas Psicométricas para discutir, especificamente, cada uma das dimensões que seriam avaliadas pelo instrumento e, então, construir os itens que comporiam cada uma delas, dentro de um formato de afirmativas para serem apreciadas por meio de uma escala. $\mathrm{Na}$ etapa de construção dos itens, foram amplamente trabalhados em aula os critérios que a literatura especializada propõe que sejam considerados na sua elaboração. Assim, após primeira versão dos itens elaborados por cada subgrupo de alunos, estes foram avaliados pelos outros colegas, segundo regras sugeridas por Fink e Kosecoff (1998) para formulação de questões fechadas. Por exemplo: cuidar para que cada questão tenha significado para o respondente, usar linguagem clara e básica, elaborar questões de modo mais concreto possível, evitar palavras ou frases indutivas, não fazer questões pessoais e observar que cada questão aborde somente um conteúdo.

\section{4a etapa - Definição da escala de respostas}

A partir da elaboração dos itens, passou-se a discutir quais seriam as melhores alternativas de respostas. Concluiu-se que, para este instrumento, o ideal seria uma escala do tipo Likert, de seis pontos. Os pontos ficaram assim classificados: (1) totalmente insatisfeito(a), (2) muito insatisfeito(a), (3) insatisfeito(a), (4) satisfeito(a), (5) muito satisfeito(a) e (6) totalmente satisfeito(a).

Segundo Pasquali (1996), a escala Likert é a mais utilizada nas Ciências Sociais, especialmente em levantamentos de atitudes, opiniões e avaliações. Esta forma de medição se caracteriza por apresentar um determinado número de alternativas em que o 
respondente deve julgar um enunciado. Geralmente, nas escalas do tipo Likert, utilizam-se números ímpares nas alternativas. No entanto, optou-se por construir uma escala com seis pontos na tentativa de impedir que o(a) respondente, ao marcar o ponto do meio da escala, não se posicionasse ante o item a ser avaliado.

\section{$5 \underline{a}$ etapa-Construção dos itens de identificação}

Optou-se por inserir no instrumento poucos itens relativos a dados de identificação a fim de preservar o máximo possível o anonimato dos respondentes. O cuidado com o sigilo, neste caso, era extremamente importante para que os alunos respondentes se sentissem absolutamente livres para emitir suas respostas, tornandoos mais participativos e disponíveis e, por conseguinte, gerando uma avaliação mais fidedigna. Contudo, a opção pelo cuidado com o sigilo dos participantes impossibilitou o levantamento de dados demográficos e maior detalhamento para a caracterização da amostra.

\section{6a etapa - Elaboração da primeira versão do instrumento}

Reunindo os materiais elaborados nas etapas três, quatro e cinco, montou-se a primeira versão do instrumento. Além de responderem, eles mesmos, o instrumento, a turma de alunos da disciplina Técnica Psicométricas revisou criteriosamente esta primeira versão, realizando pequenos ajustes que visavam a uma melhor padronização da linguagem empregada na redação dos itens.

\section{7 a etapa - Validação de conteúdo e aparente}

Submeteu-se a primeira versão do instrumento a uma avaliação dos professores que compõem o Programa. Neste sentido, Arreola (1995) ressalta a necessidade de um sistema de avaliação ser funcional. Isto é, além de produzir dados estatísticos e psicométricos, estes devem ser aceitos pelos alunos e pelo corpo docente. Desta forma, a idéia foi realizar uma validação de conteúdo pelos professores, buscando determinar a apropriação dos itens e dimensões do instrumento.

A validação de conteúdo não é determinada estatisticamente, mas é o resultado do julgamento de diferentes juízes ou pessoas de reconhecido saber na área sobre o conteúdo que está sendo medido, os quais analisam a representatividade dos itens em relação aos conceitos avaliados (Anastasi \& Urbina, 2000; Fachel \& Camey, 2000; Pasquali, 2001). Para esses autores, o instrumento possui validade de conteúdo se os itens que o constituem são representativos do universo que ele pretende representar e, além disso, se são, do ponto de vista teórico, indicadores da variável a ser medida.

Além da validade de conteúdo propriamente dita, um outro tipo de validade também relaciona-se à análise dos itens: trata-se da validade de face ou validade aparente, a qual refere-se ao que o teste mede superficialmente. Ou seja, diz respeito à linguagem e à forma em que o conteúdo está sendo apresentado (Anastasi \& Urbani, 2000; Fachel \& Camey, 2000; Latiesa, 1996). Nesta perspectiva, no presente estudo, os 14 professores do programa de pós-graduação atuaram como juízes, fazendo uma avaliação crítica da linguagem e da forma de apresentação dos itens do instrumento, verificando se eram pertinentes à avaliação de um programa de pós-graduação e se contemplavam os pontos necessários para essa avaliação.

Com a finalidade de concluir o processo de validação aparente e de conteúdo do instrumento, alunos da disciplina Técnicas Psicométricas aplicaram o instrumento em um colega da pós-graduação, num total de sete respondentes ( 2 doutorandos, 5 mestrandos). Solicitou-se que avaliassem a linguagem e a forma de apresentação dos itens do instrumento, bem como a escala de resposta. Os pareceres desses respondentes indicaram que o instrumento era adequado aos objetivos a que se propunha, sendo o formato da escala e a formulação dos itens elementos facilitadores para sua compreensão e preenchimento.

\section{8 etapa - Estudo-piloto}

Decidiu-se fazer um estudo-piloto envolvendo todos os alunos matriculados no segundo semestre de 2002 no Programa de Pós-Graduação em Psicologia da PUCRS. Para tanto, seguiram-se os procedimentos de coleta de dados exatamente na forma como pretendiase que fossem realizados no estudo definitivo: durante o processo de matrícula, todos os alunos recebiam uma cópia do instrumento, a qual deveria ser respondida de forma anônima e depositada em uma urna lacrada na secretaria do programa. Essa foi uma medida adotada para manter o critério de confidencialidade e anonimato dos dados, deixando os alunos mais à vontade para responderem ao instrumento.

De um total de 95 alunos matriculados (33 no doutorado e 62 no mestrado), obteve-se um retorno de 52 participantes $(54,7 \%)$. Dentre os principais resultados do estudo-piloto, com relação ao instrumento, podem-se destacar os seguintes:

a) Avaliação quantitativa dos dados: com o objetivo de aprimorar a versão final do instrumento, analisaramse os itens que apresentaram três ou mais respostas nulas no estudo-piloto, avaliando-se a formulação e pertinência da questão dentro do instrumento, integrando esta análise aos resultados trazidos na avaliação qualitativa;

b) Avaliação qualitativa: analisaram-se todas as sugestões trazidas pelos respondentes no espaço deixado para comentários no final do instrumento, dentre as quais destacou-se a necessidade de tornar algumas questões mais compreensíveis. Além disso, retirou-se a questão que continha a avaliação individual das disciplinas

Psico-USF, v. 10, n. 2, p. 141-147, jul./ dez. 2005 
oferecidas, a fim de anular qualquer possibilidade de identificação dos participantes através de informações sobre quais disciplinas foram cursadas pelo respondente no período avaliado.

\section{9a etapa - Estudo definitivo}

O estudo para coleta de dados numa amostra definitiva contou com a participação de 63 alunos, do total de 96 matriculados, correspondendo a $65 \%$ do total. Destes, 46 eram mestrandos e 17 eram doutorandos. A sistemática de coleta de dados foi idêntica à do estudopiloto. Cabe ressaltar que somente as autoras do presente artigo tiveram acesso direto aos instrumentos respondidos e foram responsáveis pelo tratamento e análise dos resultados.

Nesta etapa, buscou-se avaliar algumas características psicométricas do instrumento, em especial de consistência interna, além de uma avaliação global (considerando tanto as análises quantitativas quanto qualitativas).

O problema da fidedignidade aparece na literatura sob uma série de outras denominações, tais como precisão, constância, consistência interna, confiabilidade, estabilidade, confiança ou homogeneidade (Pasquali, 1996). A fidedignidade faz referência à propriedade do instrumento que produz os mesmos resultados em diferentes provas. Sendo assim, esse conceito diz respeito ao problema de estabilidade no tempo e de consistência interna da escala (Latiesa, 1996).

Para medir a fidedignidade do instrumento, optou-se por utilizar o coeficiente alfa de Cronbach, que mede a consistência interna do instrumento, analisando a homogeneidade do conteúdo expresso por meio dos itens. A medida do coeficiente alfa de Cronbach é estabelecida pela correlação do item com a escala total (Fachel \& Camey, 2000). Os métodos de consistência interna têm também como objetivo identificar itens que não devem ser mantidos na escala (itens "ruins", segundo Silveira, 1993).

O coeficiente alfa de Cronbach obtido para cada uma das subescalas constituídas pelas dimensões abrangidas pelo instrumento, bem como para o instrumento como um todo, é apresentado na Tabela 2. Observa-se que na dimensão "Bolsas" não foi calculado, por aquela ser composta apenas por um item.

Tabela 2 - Índices de consistência interna (alfa de Cronbach) do instrumento de avaliação discente do programa de pós-graduação

\begin{tabular}{lccc}
\hline Dimensões & No de itens & No de casos analisados & Coef. alfa \\
\hline Linhas de pesquisa & 3 & 61 & 0,8483 \\
Orientação & 4 & 63 & 0,9280 \\
Comunicação & 3 & 63 & 0,7930 \\
Disciplinas & 8 & 51 & 0,8561 \\
Encontro científico & 7 & 59 & 0,9597 \\
Cursos de extensão & 8 & 51 & 0,8030 \\
Integração & 3 & 60 & 0,3384 \\
Intercâmbio & 8 & 49 & 0,9214 \\
Biblioteca & 6 & 62 & 0,8624 \\
Secretaria & 6 & 63 & 0,9858 \\
Salas & 23 & 60 & 0,9389 \\
Escala total & 79 & 38 & 0,9332
\end{tabular}

Como pode ser observado na Tabela 2, os índices, tanto para cada dimensão quanto para a escala como um todo, indicam um alto grau de fidedignidade do instrumento. Esta mesma análise não evidenciou a necessidade de eliminar nenhum item da escala. Além disso, a análise qualitativa das sugestões apresentadas pelos respondentes mostrou que não havia uma demanda de alteração substancial do instrumento. Assim, decidimos manter o instrumento (à disposição dos interessados no endereço para correspondência da primeira autora) como o originalmente apresentado, corrigindo nesta etapa apenas pequenos problemas de formatação. 10a etapa - Divulgação e discussão dos resultados

Todo o processo de avaliação engendrado com base no presente instrumento culminou com uma etapa fundamental de apresentação e discussão dos resultados da coleta na amostra definitiva em reunião com a presença de todo o corpo discente e docente (incluindo a coordenação do programa), e administrativo do Programa de Pós-Graduação em Psicologia, bem como da direção da Faculdade de Psicologia da PUCRS. Esta reunião, que teve a duração duas horas e trinta minutos, buscou qualificar os dados quantitativos coletados com os alunos mediante a participação de todos os diferentes segmentos do programa, trazendo maior legitimidade às 
informações coletadas. Daí estabeleceram-se propostas de seguimento das questões assim levantadas, dentro do princípio que a avaliação institucional deve ter um caráter emancipatório e gerador de mudanças.

\section{Considerações finais}

Dentre os inúmeros desafios enfrentados para elaboração desta tarefa, que tinha como objetivo principal experimentar o processo de construção de um instrumento de pesquisa, dois merecem destaque. $\mathrm{O}$ primeiro foi a obtenção, como resultado do trabalho, de um instrumento de pesquisa para a avaliação discente da pós-graduação. Para isso, foi necessário percorrer todos os passos indicados na literatura especializada como necessários para obtenção de um material válido e fidedigno. O segundo foi a elaboração de um instrumento de avaliação de programas de pós-graduação que pudesse ser utilizado por outras instituições e que contribuísse para o aprimoramento e a qualificação do ensino em nosso país. A avaliação interna do programa encontra ressonância nos esforços que vêm sendo realizados pela CAPES (Coordenação de Aperfeiçoamento de Pessoal de Nível Superior) para a qualificação do ensino de pósgraduação no Brasil, sendo as dimensões avaliadas no instrumento baseadas nos critérios daquela instituição.

Cabe ainda ressaltar que o Programa de PósGraduação em Psicologia da PUCRS, desde a conclusão do presente estudo, tem se valido do instrumento de avaliação discente como um dos importantes componentes para a realização da sua avaliação anual. A sistemática que tem sido empregada envolve a aplicação do instrumento quando os alunos fazem a sua matrícula para o segundo semestre letivo, possibilitando, assim, que todos os respondentes tenham, no mínimo, seis meses de vivência nos cursos de mestrado ou doutorado. Os dados são coletados seguindo a mesma estratégia que já foi utilizada desde o estudo-piloto (acima relatada). Os resultados da avaliação discente têm sido apresentados e discutidos numa reunião anual com todos alunos, professores e funcionários (conforme procedimentos descritos na 10 Etapa). Busca-se, com isso, enriquecer os dados quantitativos fornecidos pelo instrumento com apreciações de cunho qualitativo que remetem tanto às dimensões abrangidas no instrumento quanto a qualquer outro aspecto que os participantes da referida reunião julgarem relevantes. Assim, produzemse as condições necessárias para gerar uma avaliação sistemática e participativa de todos os segmentos que compõem o programa, além de um mecanismo de avaliação progressiva e comparativa ao longo dos anos.

Ambiciona-se, pela divulgação do instrumento elaborado no Programa de Pós-Graduação em Psicologia da PUCRS, atingir outras instituições no país a fim de que estas possam também contar com um sistema que propicie aos discentes avaliar os cursos nos quais estão inseridos. Uma possível limitação do presente instrumento foi o fato de ter sido desenvolvido com base em apenas um programa de pós-graduação. Sem dúvida, cada programa deverá adequar o instrumento a sua realidade, uma vez que existem diferenças de funcionamento entre as instituições. Cabe destacar que esta é uma proposta inicial na tentativa de aprimorar ainda mais o sistema avaliativo existente nos programas de pós-graduação brasileiros mediante a inclusão da apreciação discente neste sistema. Entendemos que novos estudos valendose deste instrumento deverão buscar outras dimensões de validação do mesmo, além de realizar aprimoramentos possíveis tanto em relação aos itens, quanto na ampliação das dimensões avaliadas. Uma outra limitação do instrumento é o fato de não incluir uma avaliação discente individualizada de cada disciplina. Este tipo de avaliação da disciplina não foi possível de ser integrado nesta versão do instrumento, por a questões relacionadas à preservação do anonimato dos participantes, visto que o número de alunos por disciplina é usualmente pequeno (entre sete e quinze alunos matriculados por disciplina). Ainda que já existam alguns esforços preliminares tendo em vista adaptar instrumentos de avaliação discente do professor da disciplina (ver Instrumento de Avaliação Docente e do Curso, Stein, 2000), futuros estudos poderão atender a esta importante dimensão de avaliação discente.

Ademais, esperamos, com este trabalho, poder auxiliar outros pesquisadores que estejam envolvidos no processo de construção de seu instrumento de pesquisa.

O presente trabalho, que iniciou como uma atividade dentro de uma disciplina do Programa de PósGraduação em Psicologia da PUCRS, foi sendo ampliado de modo a constituir-se também em uma prática de pesquisa. Assim, foi possível ir além dos objetivos propostos inicialmente pela disciplina. Acredita-se que o processo de desenvolvimento do instrumento aqui apresentado foi além da obtenção de um instrumento válido e fidedigno para a avaliação discente de um programa de pósgraduação, pois logrou também integrar três etapas importantes para a aprendizagem em pós-graduação: o ensino (construção do instrumento), a pesquisa (avaliação discente do Programa) e a extensão (possibilidade de o instrumento ser utilizado por outros programas).

\section{Agradecimentos}

Agradecemos a colaboração valiosa dos demais colegas da disciplina Técnicas Psicométricas, bem como dos participantes da pesquisa. Nossos agradecimentos à Coordenação do Programa de Pós-Graduação em 
Psicologia da PUCRS, que incentivou a criação deste instrumento, facilitando nossa coleta de dados.

\section{Referências}

Anastasi, A. \& Urbina, B. S. (2000). Testagem psicológica. Porto Alegre: ArtMed.

Arreola, R. A. (1995). Developing a comprehensive faculty evaluation system. EUA: Anker.

Both, I. (1997). Avaliação institucional: agente de modernização administrativa e da educação. Revista da Rede de Avaliação Institucional da Educação Superior, 2(5), 33-42.

Devellis, R. F. (2002). Scale development: Theory and applications. Newbury Park, CA: Sage.

Fachel, J. \& Camey, S. (2000). Avaliação psicométrica: a qualidade das medidas e o entendimento dos dados (pp. 158-170). Em J. A. Cunha e cols. Psicodiagnóstico. (v. 5, ed. rev.) Porto Alegre: Artmed.

Fink, A. \& Kosecoff, J. B. (1998). How to conduct surveys: A step by step guide. 2nd. ed. Thousand Oaks, CA: Sage.

Latiesa, M. (1996). Validez e fiabilidad de las observaciones sociologicas. Em F. M. Garcia, J. Ibáñez \& F. Alvira (1996). El analisis de la realidad social: métodos e técnicas de investigación. 2a ed. (pp. 335-364). Madri: Alianza.
Pasquali, L. (1996). Teoria e métodos de medida em ciências do comportamento. Brasília: Laboratório de Pesquisa em Avaliação e Medida da Universidade de Brasília.

Pasquali, L. (2001). Técnicas de Exame Psicológico-TEP. Brasília: Laboratório de Pesquisa em Avaliação e Medida da Universidade de Brasília.

Pereira, J. (1997). Avaliação institucional: objetivos e critérios. Revista da Rede de Avaliação Institucional da Educação Superior, 2(5), 25-32.

Silveira, F. L (1993). Validação de testes de papel e lápis. Material preparado para a II Escola LatinoAmericana sobre Pesquisa em Ensino de Física. Porto Alegre, RS.

Spagnolo, F. (2000). Avaliação do ensino superior; dez lições da Inglaterra. Infocapes - Boletim Informativo da CAPES, 8, (1), 6-44.

Stein, L. M. (2000). Adaptação do CIEQ (Aleamoni, 1975) - Instrumento de Avaliação Docente e do Curso. Manuscrito não publicado.

Vasquez, A. (2002). Diretor de avaliação (1997-2002). Infocapes-Boletim Informativo da CAPES, 10(4), 25-30.

Recebido em novembro de 2004 Reformulado em junbo de 2005 Aprovado em setembro de 2005

Sobre as autoras:

Lilian Milnitsky Stein é docente do Programa de Pós-Graduação em Psicologia da PUCRS, diretora da Faculdade de Psicologia da PUCRS, Ph.D. em Psicologia pela University of Arizona, EUA, bolsista produtividade em pesquisa do CNPq.

Denise Falcke é psicóloga, doutora em Psicologia (PUCRS), professora e coordenadora do curso de Psicologia da Faculdade Faccat (Faculdade de Taquara-RS).

Juliana Carmona Predebon é psicóloga, doutora em Psicologia (PUCRS) e professora universitária (ULBRA).

Kátia Bones Rocha é psicóloga graduada pela PUCRS, mestre em Psicologia Social e da Personalidade por esta mesma instituição (bolsista do CNPq) e aluna da Residência Integrada em Saúde no Ambulatório de Dermatologia Sanitária do Rio Grande do Sul.

Luciana Moreira de Ávila é graduada em Psicologia pela UCPEL, mestre em Psicologia Social e da Personalidade pela PUCRS (bolsista do CNPq). Atualmente trabalha com Neuropsicologia e Avaliação Neuropsicológica.

Mariana Porto Ruwer de Azambuja é psicóloga graduada pela PUCRS, mestre em Psicologia Social e da Personalidade por esta mesma instituição (bolsista do CNPq) e aluna da Residência Integrada em Saúde no Centro de Saúde-Escola Murialdo/Ênfase em Atenção Primária à Saúde. 


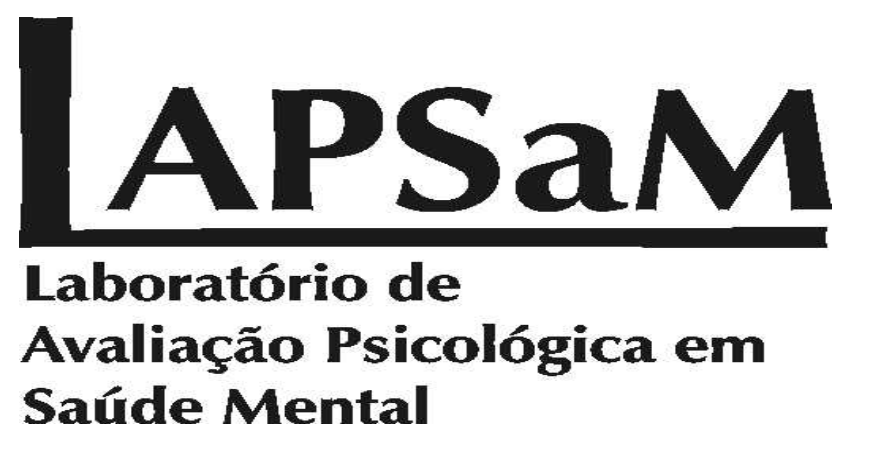

O LAPSaM desenvolve estudos sobre avaliação psicológica em contextos de saúde mental, com a validação de instrumentos e suas aplicações. Os estudos têm enfocado instrumentos psicométricos, neuropsicológicos e técnicas projetivas, apoiando-se em métodos quantitativos e qualitativos. Participam do laboratório alunos de pós-graduação e de iniciação científica.

\section{Professores responsáveis:}

Alessandra Gotuzo Seabra Capovilla alessandra.capovilla@saofrancisco.edu.br

Anna Elisa de Villemor Amaral

anna.villemor@saofrancisco.edu.br

Cláudio Garcia Capitão

claudio.capitao@saofrancisco.edu.br

Makilim Nunes Baptista

makilim.baptista@saofrancisco.edu.br 